TECHNICAL NOTE

\author{
K.L. Weiss \\ C.R. Richards \\ D. Sun \\ J.L. Weiss
}

\section{Subminute Fat-Water-Separated Dual-Echo Automated Spine Survey Iterative Scan Technique}

SUMMARY: We developed and tested an automated sub-minute 3D dual-echo MR imaging technique producing fat-water color-encoded labeled images of the entire spine. Twenty-one subjects were scanned with the 2-point Dixon technique utilizing 2 contiguous 21-22 second breath-hold sagittal acquisitions. Fourteen alternating subject scan sessions were achieved in 58 minutes. In all cases, fat-water separation was homogenous over the $70 \mathrm{~cm}$ FOV; in 2 lower stations fat/water assignments were reversed. Rapid automated fat-water decomposition spine screening is a promising technique.
C onventional MR imaging spine examinations lack standardization and are technologist-dependent. They are costly and time-consuming, often requiring 1-2 hours to image the entire spine fully. Additionally, spinal anatomic variance and limited coverage in MR imaging studies cause difficulties in definitive numbering of vertebrae and disks, resulting in an increased risk of performing spinal intervention at an incorrect level. ${ }^{1} \mathrm{MR}$ imaging quality is also reduced in patients with back pain or spasms who cannot remain motionless during image acquisition. To address these concerns, Weiss et $\mathrm{al}^{2}$ developed the automated spine survey iterative scan technique (ASSIST) using out-of-phase fast gradientecho (FGE) sequencing. ASSIST autoimages and labels the entire spine with an acquisition time of 42 seconds, limiting the potential for motion artifacts and providing definitive numbering of vertebrae and disks.

Although most promising, particularly as a scout localizer, ASSIST, as initially presented, had some limitations. The technique was only tested in the adult population, applied only to 1.5T MR imaging, provided limited coverage of the spine (7 sagittal sections), and lacked optimized tissue contrast. ASSIST was subsequently modified to include autoprompted breath-hold sequencing at 3T, increased coverage (11 sections), and imaging of the pediatric population. ${ }^{3}$ To improve tissue discrimination, we integrated supplemental fast spinecho (FSE) T2- and T1-weighted iterative decomposition of water and fat with echo asymmetric and least squares estimation (IDEAL) sequencing ${ }^{4}$ into the ASSIST algorithm. The multiparametric contrast provided improved tissue characterization and pathology detection but required approximately

Received December 31, 2008; accepted after revision March 6, 2009.

From the Department of Radiology (K.L.W., D.S., J.L.W.), University of Cincinnati, Cincinnati, Ohio; University of Cincinnati College of Medicine (C.R.R.), Cincinnati, Ohio; Institute of Information Science (D.S.), College of Computer and Information Technology, Beijing Jiaotong University, Beijing. P.R. China; and Division of Research (J.L.W.), Westimage, Cincinnati, Ohio.

This work was funded in part by the State of Ohio Child Care grant; the American Cancer Society, Ohio Division, Inc; and the University of Cincinnati Cancer Center grant. Investigational IDEAL and LAVA DE software was provided by GE Healthcare.

K.L.W. and J.L.W. have proprietary interests in $\mathrm{ASSIST}^{+}$, US patent pending.

Paper previously presented at: Annual Meeting of the American Society of Neuroradiology, May 16-21, 2009; Vancouver, British Columbia, Canada.

Please address correspondence to Kenneth L. Weiss, MD, Department of Radiology, University of Cincinnati Medical Center, 234 Goodman St, PO Box 670762, Cincinnati, OH 45267-0762; e-mail: Pweisskl@uc.edu

Indicates open access to non-subscribers at www.ajnr.org

DOI 10.3174/ajnr.A1619 15-minute total acquisition time for a sagittal survey, pre- and postcontrast, and slightly longer if saturation bands to reduce motion artifacts were applied to the FSE IDEAL sequences, which, in contrast to FGE sequencing, could not be performed with the patient breath-holding. ${ }^{5}$ IDEAL ASSIST has demonstrated high sensitivity and specificity, the former potentially that exceeding positron-emission tomography for the detection of breast metastases to the spine. ${ }^{6}$

Faster acquisition fat-water-separation techniques have been recently implemented enabling breath-hold acquisitions. ${ }^{7} \mathrm{Ma}^{8}$ developed such a modified version of the 2-point Dixon technique, using a dual-echo 3D acquisition with a robust phase-correction algorithm. Although not as accurate as the higher order multiecho IDEAL with multipeak reconstruction, ${ }^{9}$ such dual-echo techniques can reliably separate water and fat, qualitatively assess fat content, and have shown particular promise in gadolinium-enhanced abdominal MR imaging. ${ }^{10}$ Applied to the spine, the 3D breath-hold technique might significantly reduce the time required for a fat-waterdecomposed survey compared with IDEAL ASSIST, ${ }^{5}$ which in turn offered considerable time savings compared with conventional MR imaging spine screening. The goal of this investigation was to develop and test a dual-echo version of ASSIST designed to reduce the time required for a total spine survey to under a minute, mitigate motion artifacts, and provide robust fat-water separation with associated software to label vertebrae and disks automatically and provide color-encoded apparent-fat signal-intensity percentage maps and color-encoded fat-water-composite images to facilitate interpretation.

\section{Materials and Methods}

\section{Subjects}

Institutional review board approval with informed consent was obtained for this prospective Health Insurance Portability and Accountability Act-complaint research study. From June 10, 2008, to September 19, 2008, 21 subjects were examined with the investigational subminute dual-echo ASSIST (ASSIST DE). There were 15 females and 6 males (age range, 13-78 years; mean age, $41.0 \pm 17.8$ years) with the ethnicities of African American, American Indian, Asian, white, and Indian represented. Fifteen subjects underwent concurrent clinical MR imaging studies of their spines and/or brains, including 9 with breast cancer, 3 with sarcoidosis, 1 with juvenile degenerative disk disease, 1 with suspected multiple sclerosis, and 1 for a suspected spinal vascular malformation. 


\section{MR Imaging}

Studies were performed on a 3T HDx MR imaging scanner with an 8-channel spine array coil and manufacturer-supplied investigational pulse sequences (GE Healthcare, Waukesha, Wis). All subjects underwent ASSIST DE, wherein their spines, from the skull base to the sacrum, were autoimaged in 2 contiguous 21 - to 22 -second $35-\mathrm{cm}$ FOV breath-hold prompted sagittal acquisitions, yielding a composite superior-inferior FOV of $70 \mathrm{~cm}$ and an anteroposterior (AP) FOV of $35 \mathrm{~cm}$. 3D fast-spoiled gradient dual-echo 2-point Dixon sequencing was performed with autocalibrating reconstruction for Cartesian sampling ${ }^{11}$ and surface coil intensity correction (SCIC) ( $\mathrm{TR}=4.77-$ $5.44 \mathrm{~ms}$, TE1 $=1.36-1.52 \mathrm{~ms}$, TE $2 \sim 2.6 \mathrm{~ms}$, flip angle $=12^{\circ}$, bandwidth $\pm 167 \mathrm{kHz}$, superior-inferior frequency encoding $=336, \mathrm{AP}$ phase encoding $=284$, zero interpolation $=2$ and section thickness $=4 \mathrm{~mm}$ with a 2 - $\mathrm{mm}$ overlap or $3 \mathrm{~mm}$ with a 1.5 -mm overlap, 32 sections respectively providing 6.2 - or $4.8-\mathrm{cm}$ right-to-left coverage). Fat-water separation was performed by the host computer by using Ma's algorithm ${ }^{8}$ as implemented by GEHC.

Seven subjects underwent ASSIST DE without contrast, 12 without (Fig $1 A$ ) and with contrast (Fig 1B), and 2 with contrast only, the latter immediately following a brain MR imaging performed without and with contrast. Two healthy volunteers, 23 and 24 years of age, from the noncontrast subset were re-imaged multiple times to determine potential ASSIST DE throughput. The first volunteer entered the magnet and ASSIST DE was performed. The volunteer then exited the magnet to be replaced by the second volunteer. On completion of the spinal survey of the second volunteer, the first volunteer then re-entered the magnet. This process, including subject loading, unloading, table translation, prescanning, scan acquisition, reconstruction, and fat-water separation by the host processor was consecutively repeated to assess potential throughput in a 1-hour time slot.

Six subjects underwent coregistered coronal and sagittal ASSIST DE sequencing. Coronal parameters were similar to those of the sagittal acquisition with exception of reducing phase encoding from 284 to 256 so that 36 rather than 32 sections could be achieved in a similar 21- to 22-second breath-hold acquisition, while increasing AP coverage to $7.0 \mathrm{~cm}$ with $4-\mathrm{mm}$ sections spaced at $2 \mathrm{~mm}$ (Fig 2). Thirteen subjects had concurrent routine clinical spine MR imaging performed, albeit limited to the region of greatest clinical interest, cervical, thoracic, or lumbar-sacral, to save time and expense. Depending on clinical indication and real-time review of ASSIST DE sequencing, additional multiplanar investigational or clinical sequences were prescribed for further evaluation of suspected pathology.

\section{Image Processing}

Spine labeling was performed with a modified version of the initial ASSIST algorithm, ${ }^{2}$ redesigned to serve a broader population, including children and a wider range of imaging parameters. ${ }^{3}$ To apply the labeling algorithm, we obtained the DICOM images and imported them into eFilm (Merge Healthcare, Milwaukee, Wis) and then exported them into Matlab 7 (MathWorks, Natick, Mass). The DICOM images exported into Matlab consisted of the fat-water-separated images provided by the host computer.

In addition to numbering of the vertebrae and disks, the fat-waterseparated images were combined to produce 2 color-encoded images. Because host computer fat/water assignments were occasionally inverted in the lower station, an ad hoc algorithm to correctly determine which image set was fat and which was water was implemented. Because skin is water-containing and subcutaneous fat is deep to the skin, the computer assigns the water label to the dataset with the more posterior autodetected edge. An apparent-fat signal-intensity percentage map was created by using the coregistered fat-water images, dividing the fat image by the sum of the fat and water images [F / $(\mathrm{F}+$ $\mathrm{W}) \times 100$ ]. The resulting percentage was then applied to the jet color map in Matlab, which ranges from blue to red, with blue being $0 \%$ fat and red being $100 \%$ fat. In areas of low signal intensity, the F / (F + $\mathrm{W})$ ratio approached $0: 0$, increasing the noise. To minimize this noise, we set the areas where fat + water was small $(<4 \%$ maximum intensity) to zero (Fig 3).

The second color-encoded image created by Matlab was a combined fat-plus-water image, with the fat being in the red scale and the water in the gray scale. The fat image was first imported into Matlab and converted to a red scale image in the RGB color space $(\mathrm{R}=$ value, $\mathrm{G}=0, \mathrm{~B}=0)$. The water image was left in the gray scale $(\mathrm{R}=\mathrm{G}=\mathrm{B}=$ value) and then added to the fat image. The resulting image consisted of the water being in the gray scale and the fat being in the red scale $(\mathrm{R}=$ water + fat, $\mathrm{G}=\mathrm{B}=$ water $)$.

\section{Image Analysis}

The images generated by the ASSIST DE technique were independently evaluated by a neuroradiologist (K.L.W.). The autolabeled vertebrae and disks were checked for concordance with the neuroradiologists' assignments. The fat-water-separated images were qualitatively evaluated to determine homogeneity of fat-water separation and accuracy of fat-versus-water labeling by the host processor. Additionally, the comparative utility of the 4 gray-scale images (in phase, out of phase, fat, and water) generated by the host computer and 2 color-encoded maps was subjectively assessed.

\section{Results}

In all 21 subjects, sagittal ASSIST DE fat-water separation was homogeneous over the entire 70-cm FOV. Although fat/water assignations were correct for all upper stations, in 2 lower stations, labeling was reversed by the host processor. Fat-water separation and assignations were less reliable in the coronal plane, with problems again limited to the lower station where 1/6 provided heterogeneous fat-water separation and $4 / 6$ swapped fat-water labels. The algorithm implemented in Matlab based on posterior edge detection, however, provided correct assignations for all sagittal ASSIST DE cases, allowing fully automated fat-water color encoding. All autonumbered (disks and vertebrae) noncontrast spine surveys were concordant with neuroradiologist's assignments. Direct autonumbering of postcontrast ASSIST DE sequences was less reliable; consequently, automated vertebral/disk labeling of such studies (Fig $1 B$ ) was based on coregistered noncontrast ASSIST DE assignments (Fig 1A).

In no instance was sagittal ASSIST DE significantly motion-degraded or was there significant interscan motion/ patient position change between the two 21- to 22-second breath-hold acquisitions. Alternating 2 healthy volunteers, we performed 14 sagittal ASSIST DE spinal surveys in 58 minutes, yielding an average subject throughput of 4 minutes 9 seconds.

The 21 subjects imaged demonstrated a variety of pathologies with ASSIST DE, which were confirmed with conventional clinical MR imaging or supplemental IDEAL sequencing when available. Three had spinal breast metastases ( 1 such subject is illustrated in Fig $1 A,-B$ ), and another subject had extensive multilevel marrow replacement with sarcoidosis (Fig 4). In the 3 patients with breast metastases, ASSIST DE 


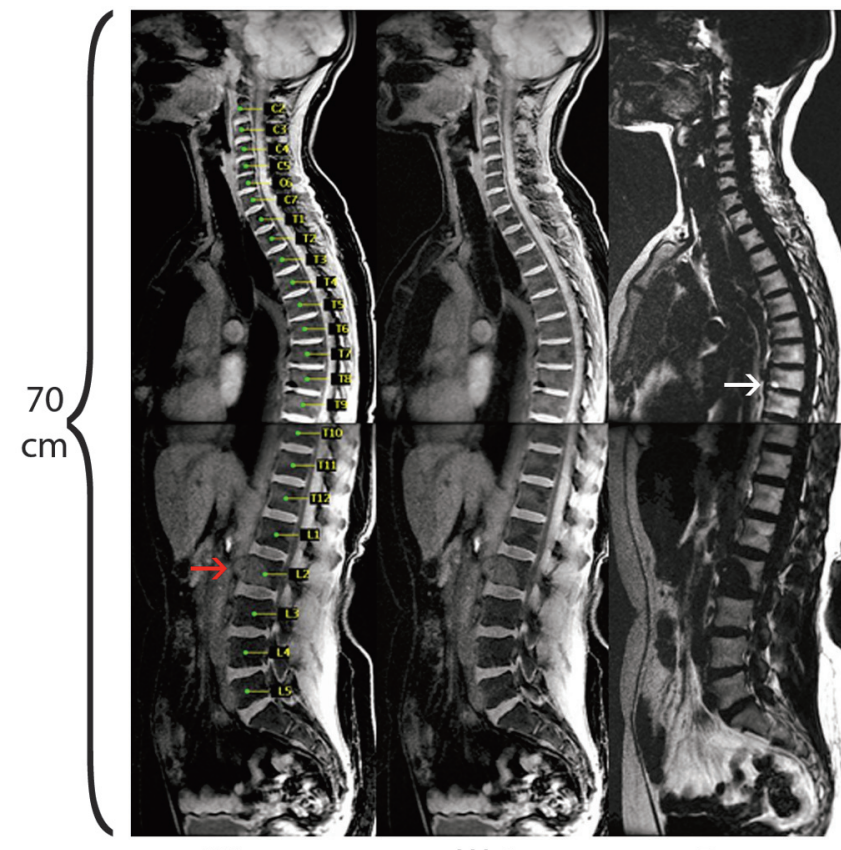

Water

A Autolabeled

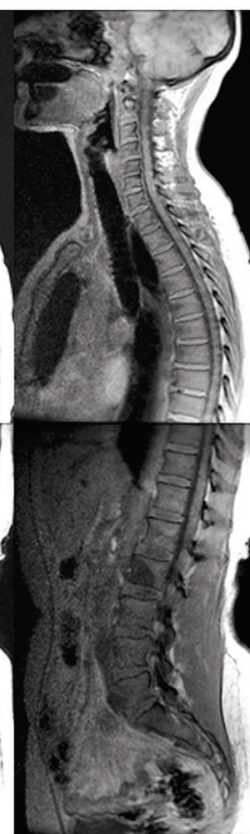

Water + Fat (In Phase)

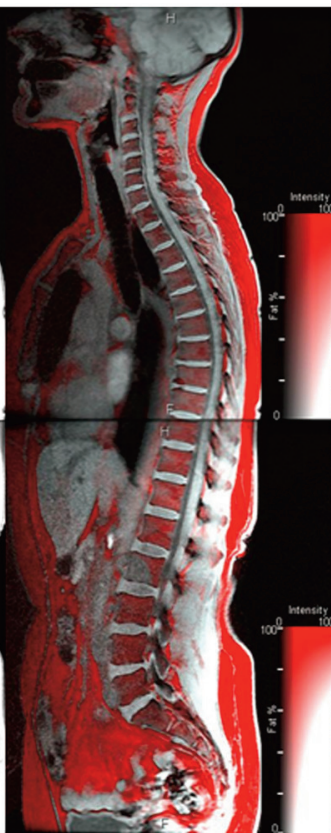

Water + Fat

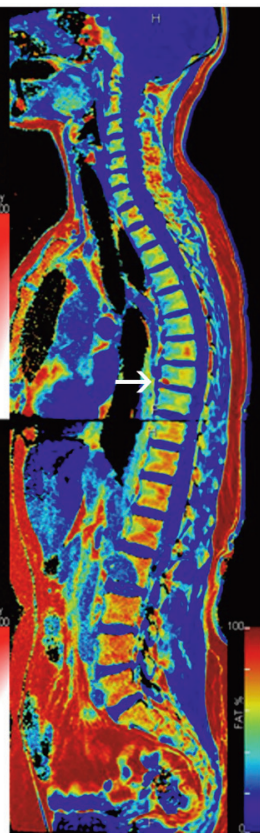

Fat \%

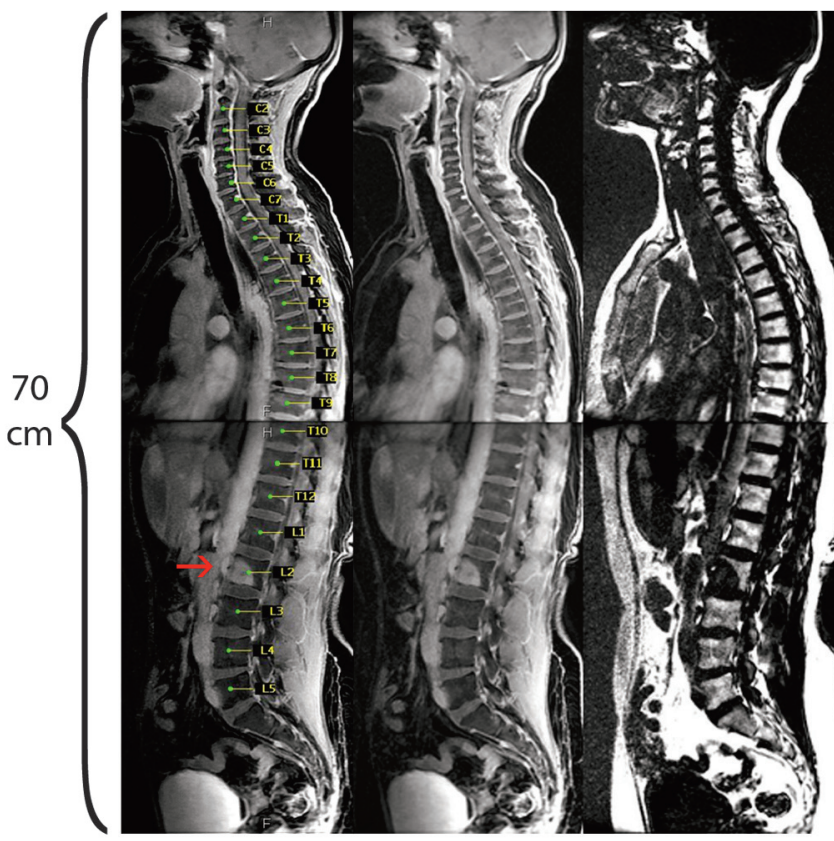

Water

Water

B Autolabeled

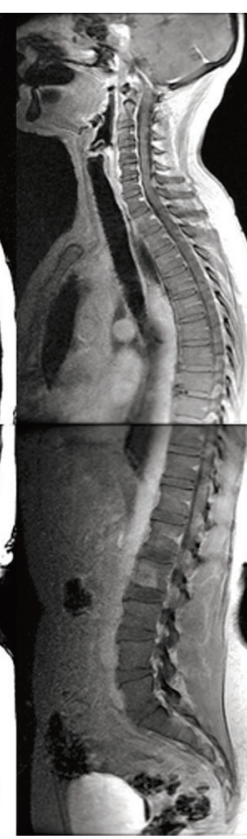

Water + Fat (In Phase)

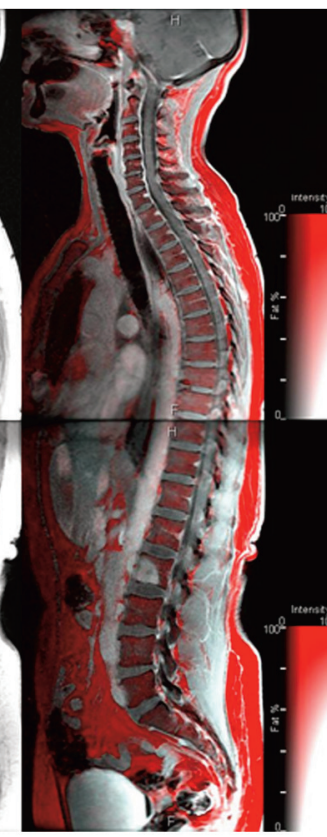

Water + Fat

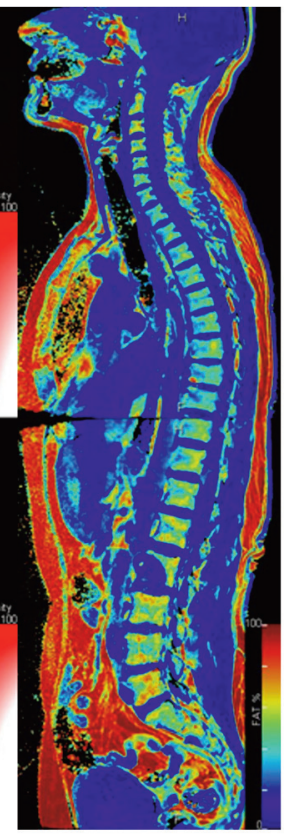

Fat \%

Fig 1. A 61-year-old woman with metastatic breast cancer. Midline sagittal ASSIST DE precontrast images $(A)$ and postcontrast images $(B)$. From left to right: autolabeled water-decomposed, non-labeled water-decomposed, fat-decomposed, water plus fat (in-phase), color-encoded water (gray) plus fat (red), and colorized apparent-fat signal percentage map. Red arrows point to solitary L2 metastasis, visible on all sequences. The metastasis replaces fat and enhances with contrast. Incidental Modic type II degenerative changes (fatty marrow conversion) involving the anterior inferior endplate $\mathrm{T} 8$ are best appreciated on the precontrast fat-decomposed and fat percentage images (white arrows). Note that the $2 \mathrm{D}$ color bar for the water plus fat images has fat percentage on the $y$-axis (red hue) and signal intensity on the $x$-axis, whereas the spectral color bar for the fat ratio image goes from low (blue) to high (red) fat percentage.

demonstrated all lesions seen by more conventional MR imaging techniques and IDEAL. However, in 1 of these patients, intravenous contrast was required to identify the smaller of 2 intradural metastases, both of which could be depicted with noncontrast FSE T2 sequencing. As with more conventional sequencing, contrast was required to appreciate the full extent of spinal disease in our sole patient with sarcoidosis. Of note, in this patient, ASSIST DE performance was comparable with that of IDEAL but better than that of conventional chemical shift fat-suppressed T1 sequencing, because the latter had some regions of inhomogeneous fat suppression.

Most subjects had some degree of degenerative disk dis- 

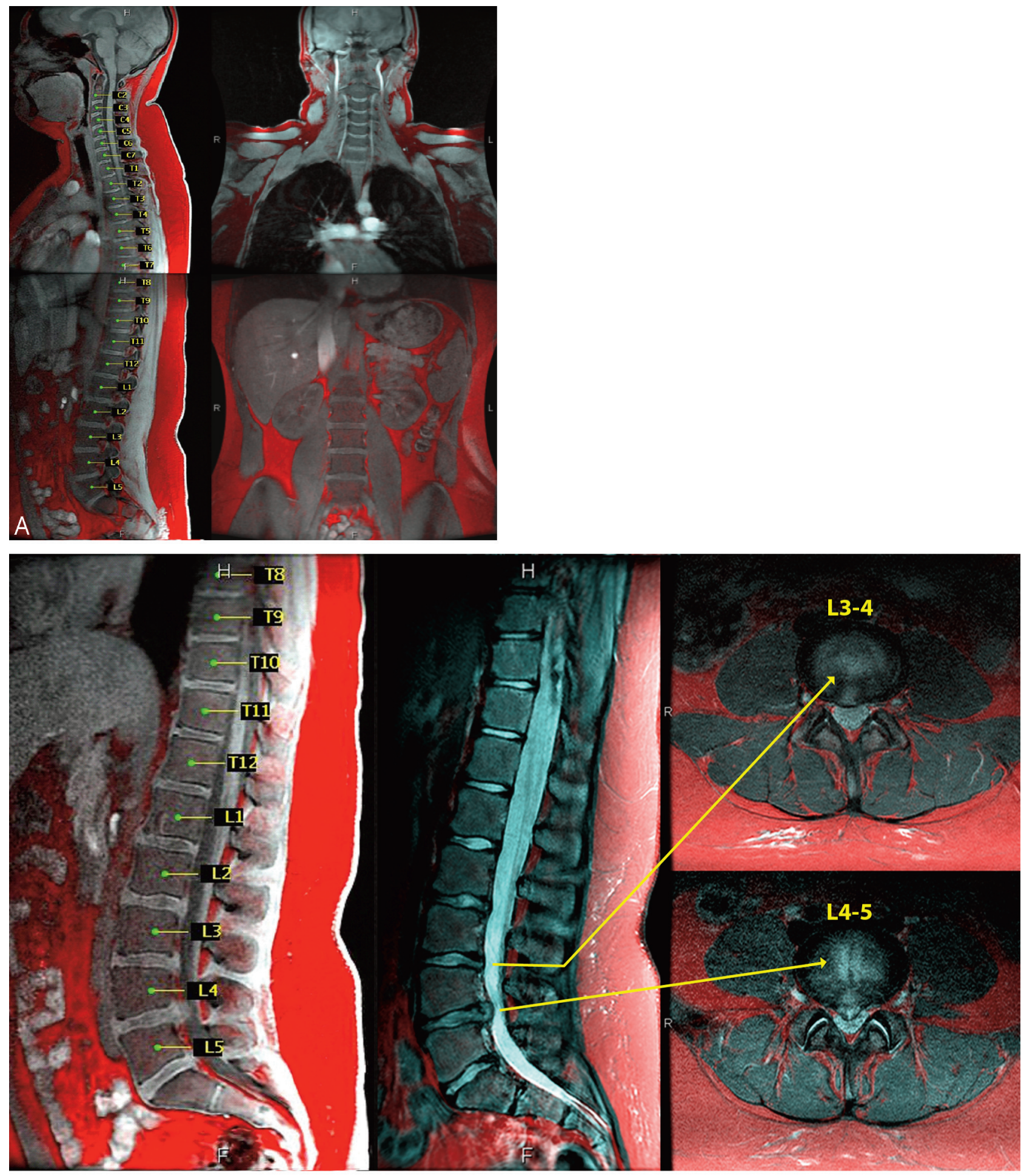

B

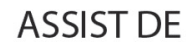

T2 FSE IDEAL

Fig 2. A 13-year-old girl with back pain. A, Color-encoded autolabeled midline sagittal ASSIST DE and corresponding coronal image. B, Color-encoded lower station sagittal ASSIST DE and corresponding 2D T2 FSE IDEAL sagittal and axial oblique images, the latter obtained for confirmation and further elucidation. Sagittal ASSIST DE demonstrates a normal cervical and thoracic spine with premature lumbar degenerative disk disease at $L 2-3$ through $L 4-5$ with mild protrusions at $L 2-3, L 3-4$, and $L 5-S 1$ and a prominent central disk herniation at $L 4-5$, T2-FSE IDEAL images were confirmatory and further elucidate the mild central protrusion L3-4 and moderate central extrusion L4-5.

ease, including one with an accelerated juvenile onset (Fig 2). When performed, coregistered coronal sequencing confirmed bone marrow abnormalities viewed in the sagittal plane and provided additional coverage of the sacrum, ileum, and retro- peritoneum. In 1 case (not illustrated), a markedly hypoplastic kidney was well demonstrated.

In all cases, pathology, particularly marrow abnormalities, could be appreciated and characterized by using the color- 


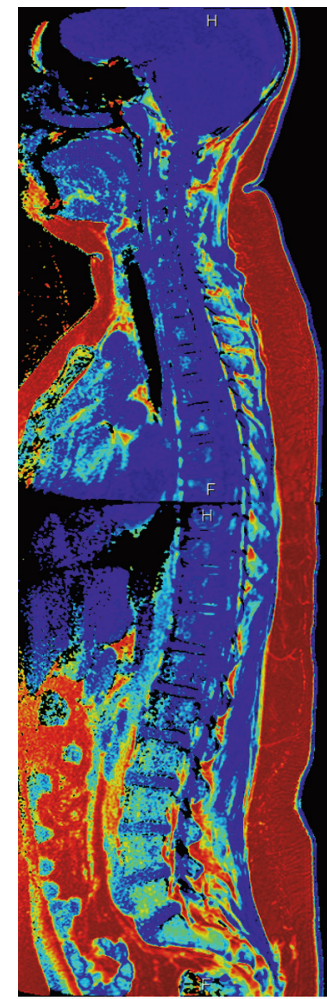

13 y.o.

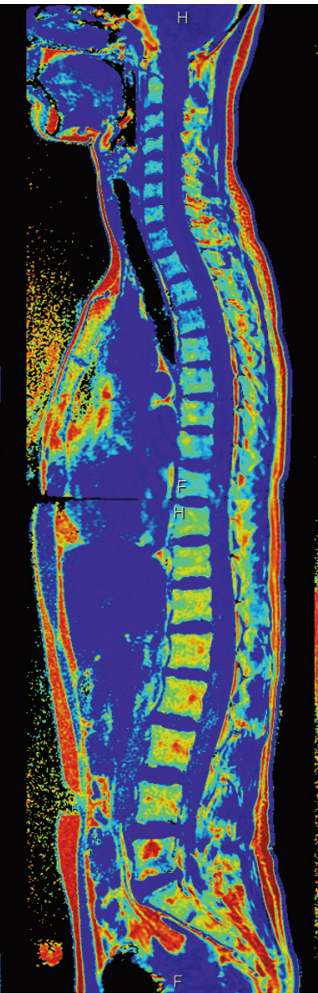

29 y.o.

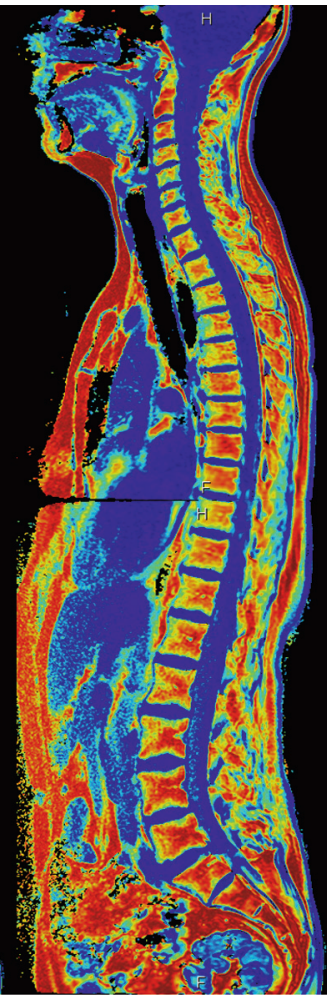

51 y.o.

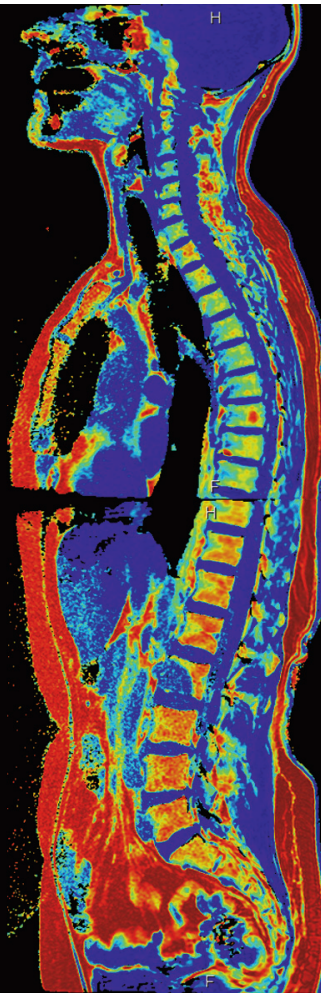

61 y.o.

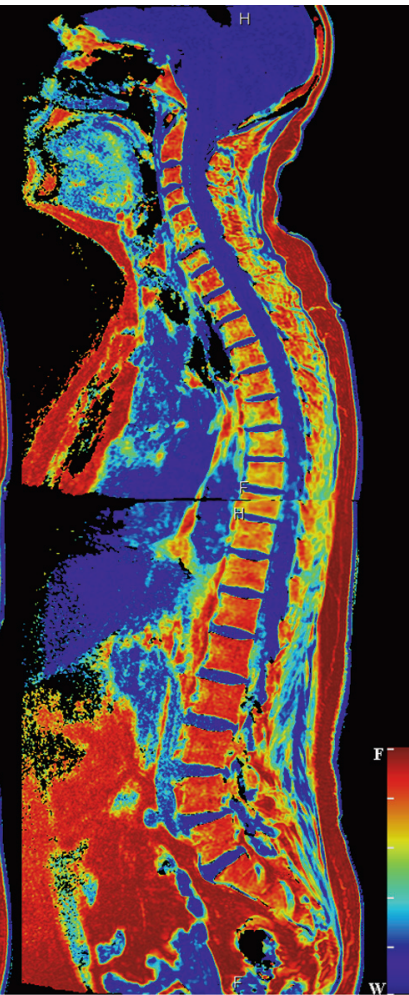

78 y.o.

Fig 3. Vertebral fat content and curvature as a function of age is demonstrated on midline sagittal ASSIST DE color-encoded apparent-fat signal-intensity percentage maps. The ages of the 5 female subjects (from left to right) are 13, 29, 51, 61, and 78 years. Note that as age increases, both kypholordosis and fatty marrow content tend to increase, the latter with a distal-to-proximal gradient. Note that the trend is not absolute because the 51-year-old woman appears to have a higher vertebral fat content than the 61-year-old woman. Additionally, percentage of fatty marrow appears relatively independent of subcutaneous fat because the youngest subject exhibits the highest subcutaneous fat volume but the lowest percentage of fatty marrow (mostly blue).
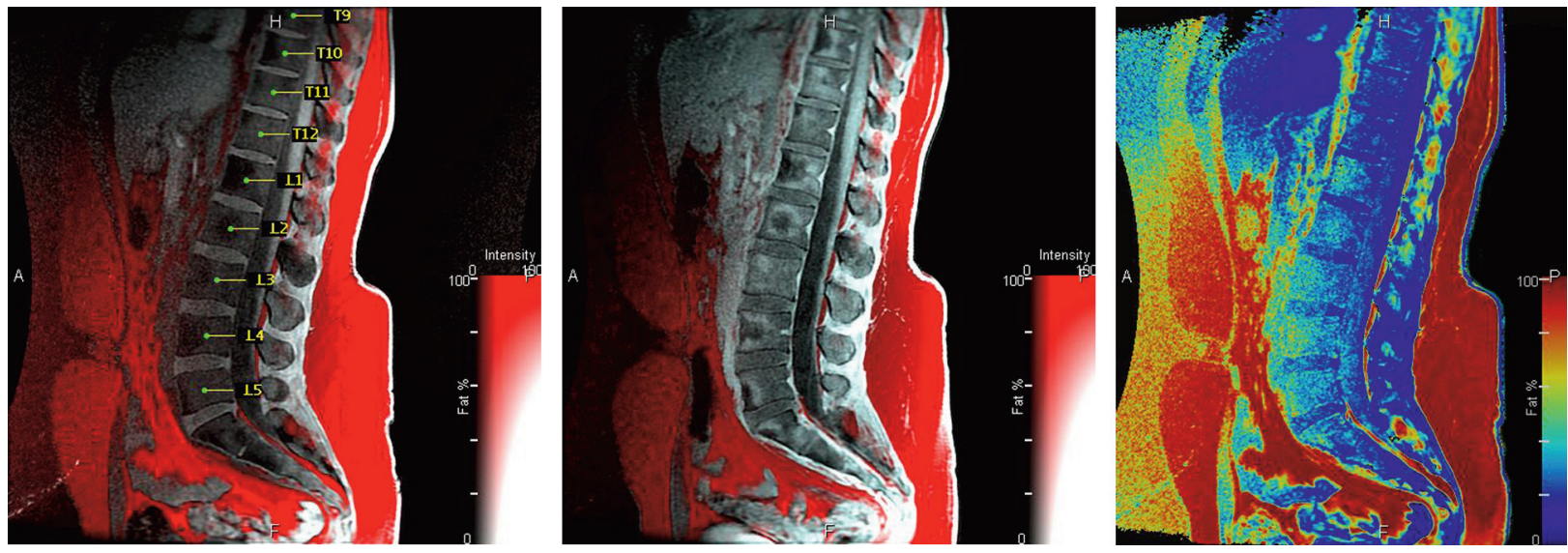

Fig 4. A 41-year-old woman with sarcoidosis. Midline lower station sagittal color-encoded ASSIST DE images from left to right: autolabeled water plus fat, postcontrast water plus fat, and precontrast apparent-fat signal-intensity percentage map. Note the diffuse fatty marrow replacement as evidenced by the paucity of reddish hue in the first 2 images and bluish color of the vertebrae on the third image. The middle image depicts heterogeneous abnormal enhancement of all visualized vertebrae related to extensive sarcoid involvement.

encoded images without reference to the 4 gray-scale series autogenerated by the host processor (Figs 1-4). Of the 2 colorencoded sequences, the gray-red scale images, which encode signal intensity (water and fat) and fat percentage in 2 dimensions, were typically favored as being the most informationrich and in all cases were preferred over the in-phase (water + fat) gray-scale images. Nonetheless, although apparent-fat/ water signal-intensity percentage can be quantified by placing regions of interest on either of the 2 autogenerated color-en- coded maps, visual assessment of fat percentage was more straightforward with the unidimensional blue-red spectral fat percentage scale. In all cases, the color-encoded apparent-fat signal-intensity percentage maps were preferred over the gray-scale fat images. Figure 3 illustrates such autogenerated apparent-fat signal-intensity percentage images derived from ASSIST DE. The images shown are from female patients ranging from 13 to 78 years of age. In addition to depicting the trend toward increasing spinal curva- 
ture with age, these images demonstrate that as age increases, the apparent fat content of the vertebral marrow tends to increase. Furthermore, apparent vertebral fat content below the $\mathrm{C} 3$ level generally increases with a rostral-tocaudal gradient, being greatest in the lumbar-sacral region. ${ }^{12,13}$

\section{Discussion}

As described, ASSIST DE provides an automated subminute sagittal survey of the entire spine with robust fat-water separation and color-encoding to facilitate tissue characterization, breath-holding for reduced motion artifacts, and computerized labeling starting from the C2-3 interspace for definitive numbering. We currently perform and recommend ASSIST $\mathrm{DE}$ as a localizer/survey/labeling sequence for all thoracic and lumbar spine studies. Although enabling time-efficient qualitative assessment of apparent-fat signal-intensity percentage as illustrated in Fig 3, due to a host of factors, including the presence of multispectral fat peaks, T2, T2*, T1, noise, coil geometry, and surface coil intensity correction effects, ASSIST DE does not permit accurate quantification of fat. ${ }^{14-17}$ Moreover, in contradistinction to IDEAL, the dual-echo technique requires additional information to identify water and fat from the 2 output images generated. ${ }^{8}$ Consequently, entire swaps of fat and water may occur as encountered in 2/42 sagittal stations with the investigational software provided (GE HealthCare), necessitating inclusion of a posterior edge (skin/water) detection algorithm to ensure correct automated fat/water assignments and color-encoding.

If desired, similar co-registered coronal ASSIST DE sequences (Fig 2B) may be obtained for further spinal evaluation, particularly in the case of scoliosis, as well as extraspinal coverage to include the sacral ala, ileum, and kidneys, the latter potentially of interest given the association of spinal and renal anomalies. ${ }^{18}$ Additionally, if they are centered slightly lower or a larger FOV ia used, the hips can be included in such a subminute supplementary coronal screen. If pathology is suggested visually or by on-line computer analysis, additional sagittal (Fig 2C) or axial oblique sequencing (Fig 2D) may be manually or autoprescribed for further evaluation and characterization while the patient is still in the magnet.

Regarding computer-assisted diagnosis, our laboratory is refining automated vertebral/disk segmentation algorithms and establishing a normative data base for ASSIST DE, to include apparent-fat signal-intensity percentage and variability as a function of age, sex, and vertebral level to supplement our morphologic data base. (Fig 3) The ultimate goal is to fully automate iterative scan prescriptions and image analysis so that if real-time computer review of the initial subminute ASSIST DE survey reveals potential pathology, appropriate sequencing for further elucidation can be autoprescribed while the patient is still in the magnet and a comprehensive report can subsequently be generated.

When a combined head-spine array coil becomes available, a complete automated neuroaxis screen should be achievable, providing rapid coverage of the brain and the entire spine in 2 or 3 sagittal 3D stations, depending on system capabilities. Although our current gradient system limits ASSIST DE sequencing to a maximum FOV of $35 \mathrm{~cm}$, newer generation $\mathrm{MR}$ imaging scanners/gradient systems can accommodate larger
FOVs so that a 90 -cm superior-inferior FOV is achievable in 2 contiguous or slightly overlapping sagittal stations as desired. Currently, the spine-labeling algorithm is semiautomated, requiring manual input of a C2-3 seed to achieve $>95 \%$ accuracy. It is hoped that inclusion of the head, which in contradistinction to the spine is a rigid body, will facilitate accurate autoidentification of the $\mathrm{C} 2-3$ interspace, obviating operator input.

Given a total acquisition time of 42-44 seconds and potential throughput at just $>4$ minutes, ASSIST DE holds promise to become a cost-effective nonionizing screening and surveillance procedure for both the adult and pediatric populations and obviates sedation for patients who cannot remain still for lengthy conventional spine MR imaging. ${ }^{3,5}$ Although 4-minute throughput was achievable with healthy volunteers in their twenties, more time will undoubtedly be required for some patients, particularly those who are not ambulatory. For patients at risk of spinal lesions such as those with known malignancy, sarcoidosis, or neurofibromatosis, ASSIST DE could serve as a baseline screening examination, similar to mammography, to detect existing lesions and increase the specificity of subsequent examinations. Automated vertebral numbering and derived color-encoded maps as described herein may facilitate interpretation of these fast but information-rich examinations. In current practice, when color-encoded images are available, only the gray-scale water and 2 color-encoded series are reviewed because the out-of-phase, in-phase (fat + water), and fat gray-scale series do not appear to provide significant additional information.

Further testing is required to determine the sensitivity and specificity of ASSIST DE without and with contrast over a large spectrum of spinal pathologies and to assess the utility of potentially complementary screening sequences such as subminute coronal ASSIST DE or sagittal T2 FSE sequencing, the latter with or without IDEAL. Last, ASSIST DE is currently investigational and will require US Food and Drug Administration/European Union approval to be used in routine clinical examinations.

In summary, ASSIST DE is a promising technique providing an automated subminute total spinal MR imaging survey with fat-water separation, vertebral/disk labeling, and colorencoding, warranting further investigation.

\section{Acknowledgments}

We thank the fine staff at UC Physicians, especially the MR technologists Tamara Davis and Elizabeth Macaluso for their helpful expertise in ASSIST DE sequence implementation. Chris Malott provided the illustrations.

\section{References}

1. Porter RW. Spinal surgery and alleged medical negligence. J R Coll Surg Edinb 1997;42:376-80

2. Weiss KL, Storrs JM, Banto RB. Automated spine survey iterative scan technique. Radiology 2006;239:255-62

3. Weiss KL, Sun D, Weiss JL. Pediatric MR imaging with automated spine survey iterative scan technique. AJNR Am J Neuroradiol 2009;30:821-24. Epub 2009 Jan 8

4. Reeder SB, Pineda AR, Wen Z, et al. Iterative decomposition of water and fat with echo asymmetry and least-squares estimation (IDEAL): application with fast spin-echo imaging. Magn Reson Med 2005;54:636-44

5. Weiss KL, Sun D, Cornelius RS, et al. Iterative decomposition of water and fat with echo asymmetric and least-squares estimation (IDEAL) automated spine 
survey iterative scan technique (ASSIST). Magnetic Resonance Insights 2008;1:1-6

6. Weiss K, Cornelius R, Lower E, et al. IDEAL ASSIST (automated spine survey iterative scan technique): metastasis detection. In: Proceedings of the $16^{\text {th }} \mathrm{Sci}$ entific Meeting and Exhibition of International Society for Magnetic Resonance in Medicine. Toronto, Ontario, Canada. May 3-9, 2008

7. Ma J. Dixon techniques for water and fat imaging. J Magn Reson Imaging 2008;28:543-58

8. Ma J. Breath-hold water and fat imaging using a dual-echo two-point Dixon technique with an efficient and robust phase-correction algorithm. Magn Reson Med 2004;52:415-19

9. Reeder SB, Robson P, Huanzhou Y, et al. Quantification of hepatic steatosis with MRI: the effects of accurate fat spectral modeling. In: Proceedings of the $16^{\text {th }}$ Scientific Meeting and Exhibition of International Society for Magnetic Resonance in Medicine. Toronto, Ontario, Canada. May 3-9, 2008

10. Low RN, Panchal N, Vu AT, et al. Three-dimensional fast spoiled gradientecho dual echo (3D-FSPGR-DE) with water reconstruction: preliminary experience with a novel pulse sequence for gadolinium-enhanced abdominal MR imaging. J Magn Reson Imaging 2008;28:946-56

11. Brau AC, Beatty PJ, Skare S, et al. Comparison of reconstruction accuracy and efficiency among autocalibrating data-driven parallel imaging methods. Magn Reson Med 2008;59:382-95
12. Kugel, H, Jung C, Schulte O, et al. Age- and sex-specific differences in the 1H-spectrum of vertebral bone marrow. J Magn Reson Imaging 2001;13: 253-69

13. Schellinger D, Lin CS, Hatipoglu HG, et al. Potential value of vertebral proton MR spectroscopy in determining bone weakness. AJNR Am J Neuroradiol 2001;22:1620-27

14. Bydder M, Yokoo T, Hamilton G, et al. Relaxation effects in the quantification of fat using gradient echo imaging. Magn Reson Imaging 2008;26:347-59

15. Liu CY, McKenzie CA, Yu H, et al. Fat quantification with IDEAL gradient echo imaging: correction of bias from $\mathrm{T}(1)$ and noise. Magn Reson Med 2007;58:354-64

16. Yu H, McKenzie CA, Shimakawa A, et al. Multiecho reconstruction for simultaneous water-fat decomposition and $\mathbf{T}^{*}$ estimation. J Magn Reson Imaging 2007;26:1153-61

17. $\mathrm{Yu} \mathrm{H}$, Shimakawa A, McKenzie CA, et al. Multiecho water-fat separation and simultaneous R2* estimation with multifrequency fat spectrum modeling. Magn Reson Med 2008;60:1122-34

18. Riccio AI, Guille JT, Grissom L, et al. Magnetic resonance imaging of renal abnormalities in patients with congenital osseous anomalies of the spine. J Bone Joint Surg Am 2007;89:2456-59 